\title{
Magnetization Reversal in Cobalt Nanocolumn Structures Obtained by Glancing Angle Deposition
}

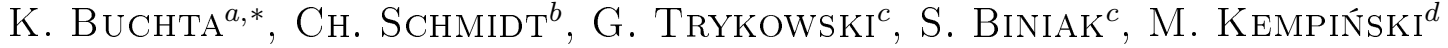 \\ AND T. LUCIŃSKI ${ }^{a}$ \\ ${ }^{a}$ Institute of Molecular Physics, Polish Academy of Sciences, M. Smoluchowskiego 17, 60-179 Poznań, Poland \\ ${ }^{b}$ University Kassel, Heinrich-Plett-Str. 40, 34132 Kassel, Germany \\ ${ }^{c}$ Nicolaus Copernicus University, J. Gagarina 11, 87-100 Toruń, Poland \\ ${ }^{d}$ Adam Mickiewicz University, Umultowska 85, 61-614 Poznań, Poland
}

\begin{abstract}
An advanced deposition technique known as glancing angle deposition was used to fabricate randomly seeded magnetic cobalt columnar nanostructures. The existence of nanocolumns was confirmed by the cross-section scanning electron microscopy. The evolution in the magnetization reversal mechanism as a function of the film thickness was investigated. The coercivity $H_{\mathrm{C}}$ and $M_{\mathrm{R}} / M_{\mathrm{S}}$ ratio (where $M_{\mathrm{R}}$ and $M_{\mathrm{S}}$ denote the remanent and saturation magnetization, respectively), derived from the magnetic hysteresis loops, are discussed as a function of the angle between the external magnetic field and the surface normal. The direction of the magnetization easy/ hard axis and the columns inclination angle were determined on the basis of the angular dependences of the $H_{\mathrm{C}}$ and the $M_{\mathrm{R}} / M_{\mathrm{S}}$. A crossover from the coherent rotation, based on the Stoner-Wohlfarth model, to the curling reversal mode was observed.
\end{abstract}

PACS: $75.70 . \mathrm{Ak}, 73.50 .-\mathrm{h}, 73.61 .-\mathrm{r}$

\section{Introduction}

The characterization and understanding of the magnetic properties of highly anisotropic ferromagnetic columnar nanostructures are interesting not only from a fundamental but also from a technological point of view owing to their potential applications in magnetic recording media, sensors and other devices [1,2]. There are still open questions about the mechanisms responsible for the magnetization reversal process and the alternation of easy axis orientation. Currently, many of the fabrication techniques are developed to manufacture micro and nanostructures with a well known location and orientation. These include electron-beam lithography [3, 4], electrodeposition on nanoporous templates [5] and epitaxial growth on single crystals [6]. The main aim of this article is to nanostructurize thin cobalt films by glancing angle deposition (GLAD) [7] method to achieve magnetic structures included vertical and tilted columns. GLAD is a physical vapor deposition method based on the self-shadowing and atomic diffusion effects at the substrate surface under oblique particle incidence (usually $>80^{\circ}$ to the substrate normal). In combination with a suitable substrate rotation and involving one or several ad-

* corresponding author; e-mail: kbuchta@ifmpan.poznan.pl justable angles between subsequent structure sections it allows to obtain more complex nanostructures including helices, chevrons and vertical posts [8]. In this article, we present the fabrication method and magnetic properties of randomly seeded cobalt posts capped with thin $\mathrm{Au}$ layer. We have investigated the thickness dependence of the magnetization reversal process, in order to find threshold thickness at which a crossover from coherent rotation mode to curling mode occurs.

\section{Experimental details}

The deposition of inclined columnar Co nanostructures was performed in an ultrahigh vacuum chamber with a base pressure better than $10^{-10}$ mbar by MBE (Prevac Precision and Vacuum Technology Molecular Beam Epitaxy system). $\mathrm{Si}(111)$ wafer, $380 \pm 25 \mu \mathrm{m}$ thick, with resistivity of more than $60 \Omega \mathrm{cm}$ was cut into $2 \times 2 \mathrm{~cm}^{2}$ pieces. The substrates were cleaned in standard procedure by immersion in acetone, ethanol, and distilled water. The layer thickness was calibrated using a quartz crystal microbalance and was ranging from 10 to $100 \mathrm{~nm}$. The angle between incident Co flux and substrate normal was set to be about $80^{\circ}$. The deposited structures were capped with $5 \mathrm{~nm}$ thick of Au layer to avoid oxidation. The deposition rates were $0.017 \mathrm{~nm} / \mathrm{s}$ and $0.014 \mathrm{~nm} / \mathrm{s}$ for $\mathrm{Co}$ and $\mathrm{Au}$, respectively. To investigate the magnetization angle dependent processes, magnetization hysteresis 
loops were taken using alternating gradient magnetometer (AGM) at room temperature with a rotatable sample holder, which allowed $360^{\circ}$ rotation with adjustable increment.

\section{Film morphology}

The characteristic feature of the sculptured thin film (STF) is strong anisotropy connected with magnetic columnar structure due to diffusion and shadowing process. The Volmer-Weber growth mode is the most important case for GLAD. Atoms will initially cover the surface in the monolayers due to the strong interaction with the substrate, but after deposition of one or several layers, islanding will occur. Systematic search showed that column tilt angle is smaller than the deposition angle. The angle correlation could be obtained by one of the fourth modes: the Nieuwenhuizen-Haanstra (tangent rule) [9], the Tait (cosine rule), the Meakin or the Lichter-Chain [10]. It is difficult to say which rule is "correct", rather experimental results tend to follow the
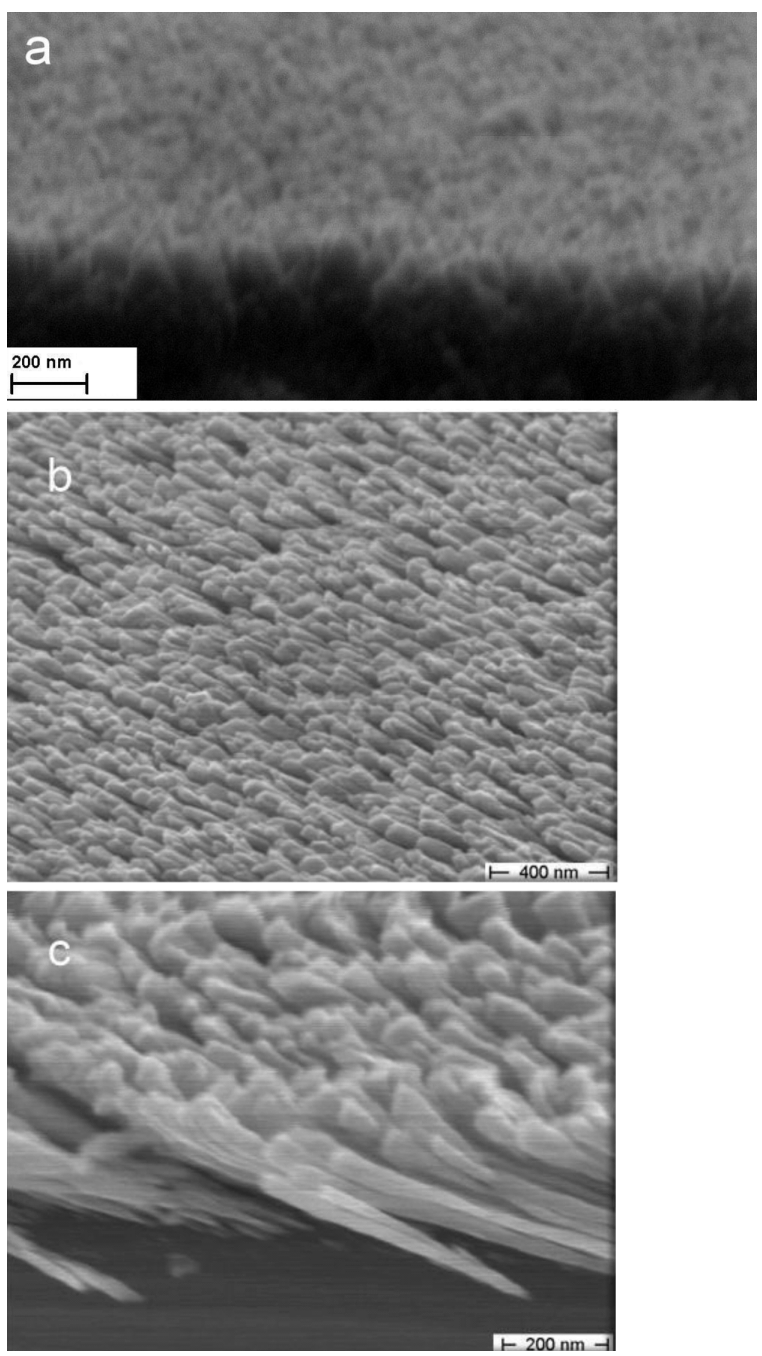

Fig. 1. SEM micrographs of Co film deposited at $80^{\circ}$ with respect to the surface normal, with thickness of $t=10 \mathrm{~nm}(\mathrm{a})$, and $t=100 \mathrm{~nm}(\mathrm{~b}, \mathrm{c})$. trends predicted by that equation. It depends on deposition condition such as class of material, pressure, degree of purity, and temperature. In order to confirm columnar structure growth, cross-section scanning electron microscopy (SEM) images were performed. Figure 1 presents an exemplary result of SEM micrographs for the samples with thickness $t=10 \mathrm{~nm}$ (a) and $t=100 \mathrm{~nm}$ $(\mathrm{b}, \mathrm{c})$. Let us note that the tilted Co columns are only observed for thicker films.

\section{Results and discussion}

The coercivity is one of the most important properties of magnetic materials from the point of view of their application and the understanding of coercivity mechanism is still a challenge for researchers. The angular dependence of $H_{\mathrm{C}}$ and $M_{\mathrm{R}} / M_{\mathrm{S}}$ ratio were extracted from those measurements. Two reversal modes have been considered as the most important: coherent and curling rotation. It is assumed that the threshold column diameter between coherent rotation and curling is $d_{\mathrm{c}}=2 \sqrt{A / M_{\mathrm{S}}}$ [11], where $A$ is the exchange constant. For column diameter of $d>d_{\mathrm{c}}$, the magnetization reversal occurs by curling as described by the Frei and Aharoni model for infinite cylinder [12]. For $d \ll d_{\mathrm{c}}$, the coherent rotation is expected as predicted by the Stoner-Wohlfarth model [13]. Furthermore for large diameter of $d \gg d_{\mathrm{c}}$, multidomain structure may form within each column thus magnetization reversal process may be determined by the domain wall motion. Figure 2 and 3 show the angular dependence of $H_{\mathrm{C}}$ and $M_{\mathrm{R}} / M_{\mathrm{S}}$ for $10 \mathrm{~nm}$ and $100 \mathrm{~nm}$ thick Co films, deposited at $80^{\circ}$.

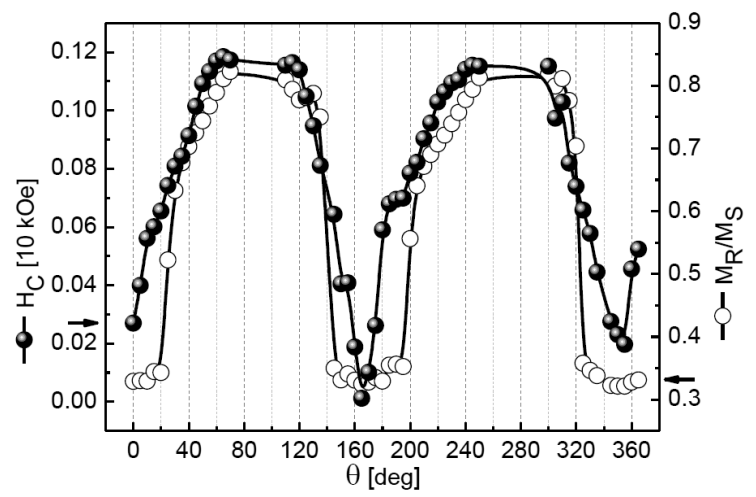

Fig. 2. Coercivity as a function of the field orientation with respect to the surface normal, for $10 \mathrm{~nm}$ thick Co film deposited at $80^{\circ}$.

For $t=10 \mathrm{~nm}$ (Fig. 2), a minimum of $H_{\mathrm{C}}$ and $M_{\mathrm{R}} / M_{\mathrm{S}}$ occurs for the field applied along surface normal $\left(0^{\circ}\right)$ and a maximum for magnetic field applied perpendicular to the surface normal $\left(90^{\circ}\right)$. These angular variations of $H_{\mathrm{C}}$ and $M_{\mathrm{R}} / M_{\mathrm{S}}$ are consistent with the coherent rotation reversal mode. For $t=100 \mathrm{~nm}$ a completely different $H_{\mathrm{C}}(\theta)$ dependence is observed (Fig. 3 ), which could be attributed to magnetization reversing in curling mode. 


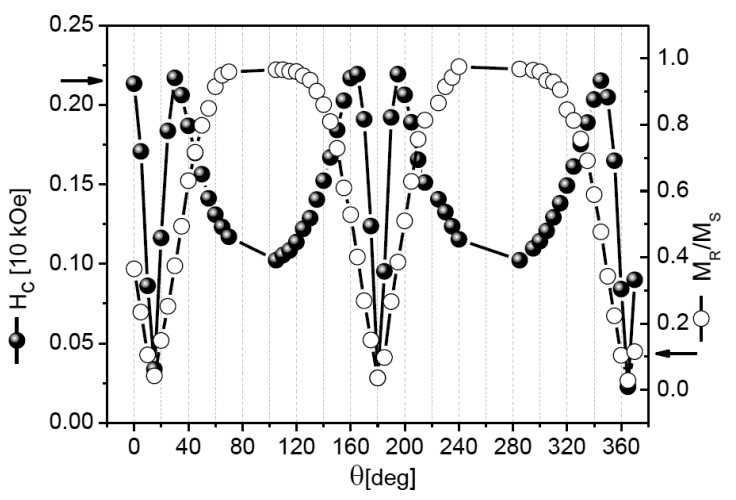

Fig. 3. Coercivity as a function of the field orientation with respect to the surface normal, for $100 \mathrm{~nm}$ thick Co film deposited at $80^{\circ}$.

Hard axis is present for field applied in orientation of $\theta=15^{\circ}, 180^{\circ}, 360^{\circ}$ (for both: curling and coherent rotation). We have modeled our experimental results using the theoretical model proposed by Aharoni and Shtrikman [14]. For nanocylinders, this model predicts the angle dependence of $H_{\mathrm{C}}(\theta)$ to follow the equation [15]:

$$
H_{\mathrm{C}}(\theta)=\frac{M_{\mathrm{S}}}{2} \frac{a(1+a)}{\sqrt{a^{2}+(1+2 a) \cos ^{2} \theta}},
$$

where $a=-1.08\left(d_{\mathrm{c}} / d\right)^{2}$.

From Fig. 4, a very good agreement is found between the measured $H_{\mathrm{C}}$ and the theoretical prediction excluding the field orientation of $\theta=90^{\circ}$ and $\theta=270^{\circ}$ which are connected with a combination of two mentioned reversal modes. Nevertheless, the curling mode dominates (see also [16]).

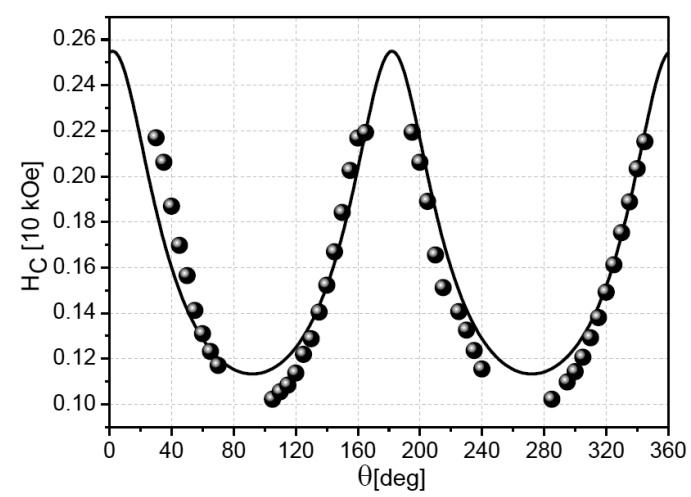

Fig. 4. Angular variation of coercivity (dots) together with theoretical prediction (solid line), based on curling mode, for $100 \mathrm{~nm}$ thick Co film deposited at $80^{\circ}$.

\section{Conclusions}

It has been shown that GLAD is a viable tool to deposit Co film consisting of columnar structures. Presented results indicate that the shapes of $H_{\mathrm{C}}(\theta)$ curves determine the magnetization reversal mechanism. We observed magnetization coherent rotation for $10 \mathrm{~nm}$ thick film and curling reversal for $100 \mathrm{~nm}$ in Co film consisting of nanocolumns. Moreover, the experimental results agrees with the theoretical modeling of the curling magnetization reversal.

\section{References}

[1] M.R. Kupsta, M.T. Taschuk, M.J. Brett, J.C. Sit, IEEE Sensors J. 9, 1979 (2009).

[2] R.D. McMichael, C.G. Lee, J.E. Bonevich, P.J. Chen, W. Miller, W.F. Egelhoff, J. Appl. Phys. 88, 3561 (2000).

[3] W. Casey Uhlig, Jing Shi, Appl. Phys. Lett. 84, 759 (2004).

[4] A.O. Adeyeye, J.A.C. Bland, C. Daboo, D.G. Hasko, H. Ahmed, J. Appl. Phys. 82, 469 (1997).

[5] R. Lavin, J.C. Denardin, A.P. Espejo, A. Cortés, H. Gomez, J. Appl. Phys. 107, 09B504 (2010).

[6] M.O. Jensen, M.J. Brett, Appl. Phys. A 80, 763 (2005).

[7] K. Robbie, G. Beydaghyan, T. Brown, C. Dean, J. Adams, C. Buzea, Rev. Sci. Instrum. 75, 1089 (2004).

[8] Ch. Patzig, Ch. Khare, B. Fuhrmann, B. Rauschenbach, Phys. Status Solidi B 247, 1322 (2010).

[9] R.N. Tait, T. Smy, M.J. Brett, Thin Solid Films 226, 196 (1993).

[10] F. Tang, D.L. Liu, D.X. Ye, Y.P. Zhao, T.M. Lu, G.C. Wang, J. Appl. Phys. 93, 4194 (2003).

[11] S. Goolaup, N. Singh, A.O. Adeyeye, V. Ng, M.B.A. Jalil, Eur. Phys. J. B 44, 259 (2005).

[12] E.H. Frei, S. Shtrikman, D. Treves, Phys. Rev. 106, 446 (1957).

[13] E.C. Stoner, E.P. Wohlfarth, Philos. Trans. R. Soc. Ser. A (London) 240, 599 (1948).

[14] A. Aharoni, S. Shtrikman, Phys. Rev. 109, 1522 (1958).

[15] S. Goolaup, A.O. Adeyeye, N. Singh, G. Gubbiotti, Phys. Rev. B 75, 144430 (2007).

[16] G.C. Han, B.Y. Zong, P. Luo, Y.H. Wu, J. Appl. Phys. 93, 9202 (2003). 